\title{
Diversity of Secondary Metabolites from Two Antarctic Microbes Rhodococcus sp. NJ-008 and Pseudomonas sp. NJ-011
}

\author{
Cheng Wang1,2, Xiaoqing Tian¹, Qiao Yang1, Yanan Lu¹, Liyan Ma1, Hongliang Huang1, \\ Chengqi Fan ${ }^{*}$ \\ ${ }^{1}$ Key Laboratory of East China Sea \& Oceanic Fishery Resources Exploitation and Utilization, \\ Ministry of Agriculture, East China Sea Fisheries Research Institute, Chinese Academy of Fishery Sciences, \\ Shanghai, China \\ ${ }^{2}$ Shanghai Ocean University, Shanghai, China \\ Email: ${ }^{\text {fancq@ecsf.ac.cn }}$
}

Received 22 May 2014; revised 21 June 2014; accepted 4 July 2014

Copyright (C) 2014 by authors and Scientific Research Publishing Inc.

This work is licensed under the Creative Commons Attribution International License (CC BY). http://creativecommons.org/licenses/by/4.0/

c) (i) Open Access

\begin{abstract}
Diversity analysis on secondary metabolites of Antarctic microbes, Rhodococcus sp. NJ-008 and Pseudomonas sp. NJ-011, together with the structural elucidation of some purified compounds, has been carried out for understanding of their chemical constituents. The methanol extracts of Rhodococcus sp. NJ-008 and Pseudomonas sp. NJ-011 were subjected to HPLC-TOF MS test for diversity analysis on secondary metabolites, respectively. The chemical constituents of $\mathrm{NJ-011}$ are mainly $\mathrm{N}$-containing compounds including some alkaloids and short polypeptides, while those of $\mathrm{NJ}-008$ are not $\mathrm{N}$-containing ones. Three compounds were also isolated and identified from extract of $\mathrm{NJ}$ 011 by different column chromatography and preparative HPLC, and their structures were elucidated as $\beta$-carboline (1), 3-benzylhexahydropyrrolo[1,2-a]pyrazine-1,4-dione (2) and 3-isobutylhexahydropyrrolo[1,2-a]pyrazine-1,4-dione (3) by comparison of TOF MS, ${ }^{1} \mathrm{H}$ - and ${ }^{13} \mathrm{C}-\mathrm{NMR}$ data with those reported. More microbial material of Pseudomonas sp. NJ-011 should be needed for exploration of the minor constituents with complicated structures.
\end{abstract}

\section{Keywords}

Antarctic Microbes, Rhodococcus sp. NJ-008, Pseudomonas sp. NJ-011, Secondary Metabolite

\footnotetext{
${ }^{*}$ Corresponding author.
} 


\section{Introduction}

Marine microbes from Antarctic area were well-known as bioactive compounds producers. In the past few years, some Chinese scientists have done fine chemical investigation from Antarctic fungui and led to the isolation of many interesting secondary metabolites, including new structures of two epipolythiodioxopiperazines and five diketopiperazines [1], two novel polyketides [2], six new peptaibols [3], together with some known aromatic phenols [4], anthraquinone derivatives and steroids [5]. During our first trip to Antarctic Ocean in 2010, some marine microbes associated with the Antarctic krill Euphausia superba had been obtained. Among these strains, Rhodococcus sp. NJ-008 was the only representative of Rhodococcus genus, while Pseudomonas sp. NJ-011 showed antimicrobial activity against Staphylococcus aureus. In the primary chemical investigation carried out for understanding the diversity of secondary metabolites from these Antarctic microbes, we found the methanol extracts of these two strains showed abundant \& interesting ion peaks in HPLC-TOF MS tests. We herein report the diversity analysis on secondary metabolites of these two microbes, and the purification and structural elucidation of three compounds from Pseudomonas sp.NJ-011.

\section{Experimental Section}

\subsection{General Procedures}

All common solvents used were of anal. grade (Shanghai Chemical Plant). Solvents for HPLC and HPLC-TOF MS: Methanol and acetonitrile (Tedia, USA). Thin-layer chromatography (TLC): pre-coated silica gel GF254 plates (Qingdao Haiyang Chemical Co. Ltd.). Column chromatography (CC): HP-20 macroporos resin (Mitsubishi Chemical Industries Co., Ltd.) and Sephadex LH-20 (Pharmacia Biotech, Sweden). Semi-preparative HPLC was performed on a Shimadazu LC-20AT pump equipped with a Shimadazu SPD-M20A PDA detector and a YMC-Pack ODS-AQ column $(250 \times 10 \mathrm{~mm}$, S-5 $\mu \mathrm{m}, 12 \mathrm{~nm})$, flow rate: $2.5 \mathrm{~mL} \cdot \mathrm{min}^{-1}$. NMR spectra were recorded on a Bruker AM-400 spectrometer; $\delta$ in ppm rel. to Me4Si, J in Hz. HR-ESI-MS were carried out on a Agilent 1290 HPLC-6224 TOF MS instrument; in m/z (rel. \%).

\subsection{Bacterial Source}

Rhodococcus sp. NJ-008 and Pseudomonas sp. NJ-011 were isolated from the Antarctic krill Euphausia superba, collected in Jan 2010 in FAO 48.1 area, and were identified by Dr. Yang Qiao. The voucher specimens (No. DHS-NJ-008 and DHS-NJ-011) were deposited in East China Sea Fisheries Research Institute, Chinese Academy of Fishery Sciences.

\subsection{Cultivation and Extraction}

Rhodococcus sp. NJ-008 in 2.0 L R2A liquid medium was shared with three $2000 \mathrm{~mL}$ Erlenmeyer flasks, and the flasks were incubated on a rotary shaker at $150 \mathrm{rpm}$ for $7 \mathrm{~d}\left(28^{\circ} \mathrm{C}\right)$. Pseudomonas sp. NJ-011 in $2.0 \mathrm{~L}$ GAUZE's Medium NO.1 also in three $2000 \mathrm{~mL}$ flasks was incubated on the same rotary shaker as one batch culture.

Pseudomonas sp. NJ-011 in 8.0 L GAUZE's Medium NO.1 in twelve $2000 \mathrm{~mL}$ flasks was incubated on the same rotary shaker at $150 \mathrm{rpm}$ for $7 \mathrm{~d}\left(28^{\circ} \mathrm{C}\right)$ as another batch culture.

The culture broth (2 L) of Rhodococcus sp. NJ-008 (or Pseudomonas sp. NJ-011) was centrifuged at $4000 \mathrm{rpm}$. The bacterial cells were frozen at $-78^{\circ} \mathrm{C}$ for $3 \mathrm{~h}$, and then were extracted with $100 \mathrm{~mL}$ acetone and $100 \mathrm{~mL}$ methanol, successively. The organic solutions were concentrated under reduced pressure to afford a residue. The supernatant was evaporated to a salty dryness in vacuum. And these two parts were combined in $100 \mathrm{~mL}$ methanol, taking $1.0 \mathrm{~mL}$ supernatant for HPLC-TOF MS tests.

The culture broth (8 L) of Pseudomonas sp. NJ-011was extracted with ethyl acetate $(1500 \mathrm{~mL})$ for three times, and the mixture was treated with ultrasonic cleaner for $30 \mathrm{~min}$ each time. The organic solutions were combined and concentrated under reduced pressure to afford a residue $(1.4 \mathrm{~g})$.

\subsection{HPLC-TOF MS test}

HPLC column: YMC ODS-AA12S03-L546WT (4.6 × $75 \mathrm{~mm}, \mathrm{~S}-5 \mu \mathrm{m}, 12 \mathrm{~nm})$; injection volume: $10 \mu \mathrm{L}$; flow rate: $0.5 \mathrm{~mL} \cdot \mathrm{min}^{-1}$; mobile phase $\left(\mathrm{CH}_{3} \mathrm{CN}\right.$ in $\mathrm{H}_{2} \mathrm{O}$ containing $\left.0.1 \% \mathrm{HCOOH}\right): 0$ - $1.2 \mathrm{~min} 5 \%, 14 \mathrm{~min} 95 \%, 17$ $\min$ 95\%, $17.5 \min 5 \%, 20 \min 5 \%$; TOF MS: positive ion mode ESI; gas temperature $340^{\circ} \mathrm{C}, \mathrm{N}_{2} 8.0 \mathrm{~L} \cdot \mathrm{min}^{-1}$, 
nebulizer 40 psi. After test, the data of ion peaks with volume $>100,000$ were extracted in TIC scan. Possible formula for each peak and the calculated exact mass for $[\mathrm{M}+\mathrm{H}]^{+}$were given by DNP database and ChemBio Draw Ultra version 12.0, respectively.

\subsection{Isolation}

Total extract (1.3 g) from $8 \mathrm{~L}$ culture broth of Pseudomonas sp. NJ-011 was absorbed by HP-20 macroporos re$\sin (600 \mathrm{~mL})$, and eluted with $\mathrm{H}_{2} \mathrm{O}(1500 \mathrm{~mL})$ and methanol/ $\mathrm{H}_{2} \mathrm{O}(10: 90,1500 \mathrm{~mL})$ to remove some salts, sugars, amino acids and proteins, and then eluted with methanol/ $\mathrm{H}_{2} \mathrm{O}(50: 50 \rightarrow$ 80:20 $\rightarrow$ 100:0, $2500 \mathrm{~mL}$ each)to afford three sections NJ-011-A (0.5 g), NJ-011-B (0.1 g) and NJ-011-C (0.4 g), successively. The section NJ-011-A was passed through a Sephadex LH-20 column, eluted with ethanol/ $\mathrm{H}_{2} \mathrm{O}$ (70:30), to yield three fractions A1 to A3. Finally, A1 were purified by repeated semi-preparative HPLC with mobile phase of $\mathrm{CH}_{3} \mathrm{CN} / \mathrm{H}_{2} \mathrm{O}-0.1 \% \mathrm{TFA}$ (25:75 $\rightarrow$ 70:30, $2.5 \mathrm{~mL} / \mathrm{min}$ ), and three compounds $\mathbf{1}$ (7.6 mg), 3 (2.3 mg), and $\mathbf{2}$ (3.5 mg) were obtained.

$\beta$-carboline (1): positive ion mode ESI $\mathrm{m} / \mathrm{z}$ : $169.0758[\mathrm{M}+\mathrm{H}]^{+}$; ${ }^{1} \mathrm{H}-\mathrm{NMR}\left(\mathrm{CD}_{3} \mathrm{OD}, 400 \mathrm{MHz}\right) \delta: 8.80(1 \mathrm{H}$, br s), $8.28(1 \mathrm{H}, b r s), 8.20(1 \mathrm{H}, d t, J=1.0,8.0 \mathrm{~Hz}), 8.10(1 \mathrm{H}, b r d, J=5.5 \mathrm{~Hz}), 7.57(1 \mathrm{H}, m), 7.56(1 \mathrm{H}, m), 7.27$ $(1 \mathrm{H}, d d d, J=3.1,5.1,8.0 \mathrm{~Hz}) ;{ }^{13} \mathrm{C}-\mathrm{NMR}\left(\mathrm{CD}_{3} \mathrm{OD}, 100 \mathrm{MHz}\right) \delta: 143.5,138.8,134.6,130.9,130.3,123.3(\mathrm{C} \times$ 2), 122.7, 121.4, 116.7, 113.3.

3-benzylhexahydropyrrolo[1,2-a]pyrazine-1,4-dione (2): positive ion mode ESI $\mathrm{m} / \mathrm{z}$ : $245.1283[\mathrm{M}+\mathrm{H}]^{+}$; ${ }^{1} \mathrm{H}-\mathrm{NMR}\left(\mathrm{CD}_{3} \mathrm{OD}, 400 \mathrm{MHz}\right) \delta: 7.28(5 \mathrm{H}, m), 4.47(1 \mathrm{H}, \mathrm{s}), 4.08(1 \mathrm{H}, m), 3.55(1 \mathrm{H}, m), 3.39(1 \mathrm{H}, m), 3.20(2 \mathrm{H}$, $m), 2.11(1 \mathrm{H}, m), 1.82(2 \mathrm{H}, m), 1.34(1 \mathrm{H}, m) ;{ }^{13} \mathrm{C}-\mathrm{NMR}\left(\mathrm{CD}_{3} \mathrm{OD}, 100 \mathrm{MHz}\right) \delta: 169.9,166.0,135.3,129.9(2 \mathrm{C})$, 128.3 (2C), 127.1, 58.4, 57.7, 44.8, 39.6, 28.4, 21.1.

3-isobutylhexahydropyrrolo[1,2-a]pyrazine-1,4-dione (3): positive ion mode ESI m/z: 211.1438 [M + H] ${ }^{+}$; ${ }^{1} \mathrm{H}-\mathrm{NMR}\left(\mathrm{CD}_{3} \mathrm{OD}, 400 \mathrm{MHz}\right) \delta: 4.27(1 \mathrm{H}, m), 4.14(1 \mathrm{H}, m), 3.54(2 \mathrm{H}, m), 2.63(2 \mathrm{H}, m), 2.32(1 \mathrm{H}, m), 2.02(1 \mathrm{H}$, $m), 1.92(1 \mathrm{H}, m), 1.67(1 \mathrm{H}, m), 1.54(1 \mathrm{H}, m), 0.99(3 \mathrm{H}, d, J=6.4 \mathrm{~Hz}), 0.98(3 \mathrm{H}, d, J=6.3 \mathrm{~Hz}) ;{ }^{13} \mathrm{C}-\mathrm{NMR}$ $\left(\mathrm{CD}_{3} \mathrm{OD}, 100 \mathrm{MHz}\right) \delta: 171.4,167.5,58.8,53.2,45.0,37.9,27.7,24.3,22.2,21.9,20.8$.

\section{Results and Discussions}

\subsection{Diversity Analysis on Secondary Metabolites}

By high-resolution HPLC-TOF MS spectra (Table 1) of methanol extract solution from Rhodococcus sp. NJ008, 7 peaks were not given possible formula by DNP database, which may indicate the constituents with new structures. The possible formulae of 25 sample peaks were obtained among the total 32 observed peaks. Except for a plasticizer (Cpd19), the possible formulae indicated 13 - 15 compounds without nitrogen in their structures, and the other 9 - 11 were N-containing compounds. There were also 2S-containing metabolites, but no halogencontaining sample peaks were found by typical MS spectra. The relative contents (Vol\%) of 14 sample peaks were higher than $2.0 \%$, including 9 compounds not bearing $\mathrm{N}$-atoms, $4 \mathrm{~N}$-containing compounds, and another one without possible formula. So the chemical constituents of Rhodococcus sp. NJ-008 are mainly non-N-containing compounds, and the $\mathrm{N}$-containing compounds showed a minor relative content.

The methanol extract solution of Pseudomonas sp. NJ-011 was also subjected to HPLC-TOF MS test. Among the total 53 observed peaks (Table 2), 10 peaks were not given possible formula by DNP database, which may indicate the constituents with new structures. The possible formulae of 43 sample peaks were obtained by highresolution HPLC-TOF MS spectra, including $29 \mathrm{~N}$-containing compounds, and the other 14 compounds without nitrogen in their structures. There were also 1 metabolite which was both S- and N-containing compound, and another compound might bear P-atom, but no halogen-containing sample peaks were found by typical MS spectra. Most sample peaks showed very low relative contents with their Vol\% much less than $1.0 \%$. Besides a plasticizer (Cpd36), there were only 10 sample peaks with Vol\% higher than 2.0\%, including $9 \mathrm{~N}$-containing compounds and another one without possible formula. So the chemical constituents of Pseudomonas sp. NJ-011 are mainly N-containing compounds, including some alkaloids and short polypeptides.

\subsection{Structural Elucidation}

Three compounds were obtained from the high polarity extract section of Pseudomonas sp. NJ-011, by different column chromatography and repeated preparative HPLC, and their structures were elucidated by comparison of 
Table 1. LC-TOF MS data of the NJ-008 methanol extract.

\begin{tabular}{|c|c|c|c|c|c|}
\hline Cpd & Possible formula & Cacld. for $[\mathrm{M}+\mathrm{H}]^{+}(\mathrm{m} / \mathrm{z})$ & Base peak $(m / z)$ & $\mathrm{RT}(\min )$ & Vol\% \\
\hline 1 & $-^{\mathrm{a}}$ & & 132.04055 & 1.677 & 1.25 \\
\hline 2 & $\mathrm{C}_{4} \mathrm{H}_{6} \mathrm{O}_{2} \mathrm{~S}_{3}$ & 182.9603 & 182.96213 & 1.679 & 1.81 \\
\hline 3 & - & & 223.98907 & 1.681 & 1.55 \\
\hline 4 & $\mathrm{C}_{8} \mathrm{H}_{6} \mathrm{O}_{3}$ & 151.0390 & 151.03505 & 1.684 & 1.9 \\
\hline 5 & - & & 265.0155 & 1.686 & 1.01 \\
\hline 6 & $\mathrm{C}_{26} \mathrm{H}_{42} \mathrm{~N}_{4} \mathrm{O}_{4}$ & 475.3279 & 475.32547 & 5.984 & 0.46 \\
\hline 7 & $\mathrm{C}_{20} \mathrm{H}_{36} \mathrm{O}_{4}$ & 341.2686 & 341.26267 & 6.665 & 9.83 \\
\hline 8 & $\mathrm{C}_{26} \mathrm{H}_{47} \mathrm{NO}_{5}$ & 454.3527 & 454.34701 & 7.044 & 4.62 \\
\hline 9 & $\mathrm{C}_{29} \mathrm{H}_{44} \mathrm{~N}_{2} \mathrm{O}_{2}$ & 453.3476 & 453.3437 & 7.279 & 4.47 \\
\hline 10 & $\mathrm{C}_{30} \mathrm{H}_{55} \mathrm{~N}_{5} \mathrm{O}_{5}$ & 566.4276 & 566.42768 & 7.298 & 1.01 \\
\hline 11 & $\mathrm{C}_{9} \mathrm{H}_{18} \mathrm{~N}_{2} \mathrm{O}$ & 171.1492 & 171.14896 & 9.521 & 4.47 \\
\hline 12 & $\mathrm{C}_{26} \mathrm{H}_{43} \mathrm{NO}_{6}$ & 466.3163 & 466.31663 & 10.182 & 0.28 \\
\hline 13 & - & & 199.18021 & 11.461 & 3.18 \\
\hline 14 & $\mathrm{C}_{13} \mathrm{H}_{24} \mathrm{~N}_{2} \mathrm{O}$ & 225.1961 & 225.19591 & 12.309 & 0.99 \\
\hline 15 & $\mathrm{C}_{19} \mathrm{H}_{22} \mathrm{O}$ & 267.1743 & 267.17183 & 15.316 & 0.45 \\
\hline 16 & $\begin{array}{c}\mathrm{C}_{19} \mathrm{H}_{26} \mathrm{O}_{6} \text { or } \\
\mathrm{C}_{15} \mathrm{H}_{30} \mathrm{~N}_{2} \mathrm{O}_{3} \mathrm{~S}_{2}\end{array}$ & $\begin{array}{l}351.1802 \\
351.1771\end{array}$ & 351.17785 & 15.513 & 0.18 \\
\hline 17 & $\begin{array}{l}\mathrm{C}_{20} \mathrm{H}_{34} \mathrm{~N}_{2} \mathrm{O}_{6} \\
\text { or } \mathrm{C}_{25} \mathrm{H}_{34} \mathrm{O}_{4}\end{array}$ & $\begin{array}{l}399.2490 \\
399.2530\end{array}$ & 399.25088 & 15.84 & 0.45 \\
\hline 18 & $\mathrm{C}_{18} \mathrm{H}_{35} \mathrm{NO}$ & 282.2791 & 282.27894 & 15.985 & 0.79 \\
\hline 19 & plasticizer $^{\mathrm{b}}$ & & 301.14378 & 16.339 & 0.67 \\
\hline 20 & $\mathrm{C}_{23} \mathrm{H}_{38} \mathrm{O}_{4}$ & 379.2843 & 379.28203 & 16.381 & 3 \\
\hline 21 & $\mathrm{C}_{18} \mathrm{H}_{37} \mathrm{NO}$ & 284.2948 & 284.29486 & 16.554 & 8.57 \\
\hline 22 & - & & 344.27943 & 16.716 & 1.03 \\
\hline 23 & $\mathrm{C}_{21} \mathrm{H}_{32} \mathrm{O}_{4}$ & 349.2373 & 349.23495 & 16.735 & 0.83 \\
\hline 24 & $\mathrm{C}_{24} \mathrm{H}_{38} \mathrm{O}_{4}$ & 391.2843 & 391.2845 & 17.96 & 4.64 \\
\hline 25 & - & & 280.26352 & 18.319 & 0.48 \\
\hline 26 & $\mathrm{C}_{24} \mathrm{H}_{40} \mathrm{O}_{4}$ & 393.2999 & 393.29749 & 18.433 & 11.2 \\
\hline 27 & $\mathrm{C}_{6} \mathrm{H}_{10} \mathrm{O}_{4}$ & 147.0652 & 147.06501 & 18.459 & 3.48 \\
\hline 28 & $\mathrm{C}_{22} \mathrm{H}_{42} \mathrm{O}_{4}$ & 371.3156 & 371.31557 & 18.461 & 9.28 \\
\hline 29 & $\mathrm{C}_{6} \mathrm{H}_{8} \mathrm{O}_{3}$ & 129.0546 & 129.05452 & 18.462 & 6.66 \\
\hline 30 & $\mathrm{C}_{14} \mathrm{H}_{26} \mathrm{O}_{4}$ & 259.1904 & 259.19034 & 18.472 & 4.25 \\
\hline 31 & - & & 402.35806 & 18.479 & 1.03 \\
\hline 32 & $\mathrm{C}_{46} \mathrm{H}_{82} \mathrm{O}_{8}$ & 763.6082 & 763.6059 & 18.521 & 6.2 \\
\hline
\end{tabular}

${ }^{\mathrm{a}}$ No possible formula given; ${ }^{\mathrm{b}}$ Ion peak of plasticizer dibutyl phthalate $[\mathrm{M}+\mathrm{Na}]^{+}$. 
Table 2. LC-TOF MS data of the NJ-011 methanol extract.

\begin{tabular}{|c|c|c|c|c|c|}
\hline Cpd & Possible formula & Cacld. for $[\mathrm{M}+\mathrm{H}]^{+}(\mathrm{m} / \mathrm{z})$ & Base peak $(\mathrm{m} / \mathrm{z})$ & $\mathrm{RT}$ (min) & Vol\% \\
\hline 1 & $\mathrm{C}_{10} \mathrm{H}_{16} \mathrm{~N}_{2} \mathrm{O}_{2}$ & 197.1285 & 197.1282 & 6.377 & 0.88 \\
\hline 2 & $\mathrm{C}_{23} \mathrm{H}_{33} \mathrm{NO}$ & 340.2635 & 340.25938 & 6.596 & 16.15 \\
\hline 3 & $\mathrm{C}_{28} \mathrm{H}_{45} \mathrm{NO}_{7}$ & 508.3269 & 508.32833 & 6.613 & 0.33 \\
\hline 4 & $\mathrm{C}_{11} \mathrm{H}_{8} \mathrm{~N}_{2}$ & 169.0760 & 169.07582 & 6.615 & 3.12 \\
\hline 5 & - & & 453.54518 & 7.025 & 0.36 \\
\hline 6 & $\mathrm{C}_{29} \mathrm{H}_{44} \mathrm{~N}_{2} \mathrm{O}_{2}$ & 453.3476 & 453.34386 & 7.025 & 7.45 \\
\hline 7 & $\mathrm{C}_{29} \mathrm{H}_{44} \mathrm{~N}_{2} \mathrm{O}_{2}$ & 453.3476 & 453.34377 & 7.261 & 6.12 \\
\hline 8 & $\mathrm{C}_{30} \mathrm{H}_{55} \mathrm{~N}_{5} \mathrm{O}_{5}$ & 566.4276 & 566.42761 & 7.287 & 1.96 \\
\hline 9 & $\mathrm{C}_{11} \mathrm{H}_{18} \mathrm{~N}_{2} \mathrm{O}_{2}$ & 211.1441 & 211.14378 & 7.465 & 0.63 \\
\hline 10 & $\mathrm{C}_{14} \mathrm{H}_{16} \mathrm{~N}_{2} \mathrm{O}_{2}$ & 245.1285 & 245.12826 & 7.747 & 0.77 \\
\hline 11 & $\mathrm{C}_{9} \mathrm{H}_{18} \mathrm{~N}_{2} \mathrm{O}$ & 171.1492 & 171.14889 & 9.449 & 7.17 \\
\hline 12 & $\mathrm{C}_{31} \mathrm{H}_{49} \mathrm{NO}_{4}$ & 500.3734 & 500.37952 & 10.786 & 0.49 \\
\hline 13 & $\mathrm{C}_{11} \mathrm{H}_{19} \mathrm{NO}$ & 182.1539 & 182.15379 & 11.277 & 0.91 \\
\hline 14 & - & & 199.1802 & 11.427 & 4.48 \\
\hline 15 & $\mathrm{C}_{13} \mathrm{H}_{24} \mathrm{~N}_{2} \mathrm{O}$ & 225.1961 & 225.19595 & 12.289 & 1.75 \\
\hline 16 & $\mathrm{C}_{9} \mathrm{H}_{19} \mathrm{NO}$ & 158.1539 & 158.15366 & 12.398 & 0.87 \\
\hline 17 & - & & 337.28505 & 12.648 & 1.13 \\
\hline 18 & $\mathrm{C}_{12} \mathrm{H}_{25} \mathrm{NO}$ & 200.2009 & 200.20057 & 14.672 & 1.57 \\
\hline 19 & $\mathrm{C}_{18} \mathrm{H}_{33} \mathrm{NO}$ & 280.2635 & 280.26367 & 14.954 & 0.77 \\
\hline 20 & $\mathrm{C}_{39} \mathrm{H}_{69} \mathrm{~N}_{5} \mathrm{O}_{9}$ & 752.5168 & 752.51521 & 15.252 & 0.17 \\
\hline 21 & $\mathrm{C}_{19} \mathrm{H}_{22} \mathrm{O}$ & 267.1743 & 267.17211 & 15.314 & 1.35 \\
\hline 22 & $\mathrm{C}_{43} \mathrm{H}_{65} \mathrm{NO}_{7}$ & 708.4834 & 708.48923 & 15.355 & 0.37 \\
\hline 23 & - & & 664.46319 & 15.453 & 0.59 \\
\hline 24 & $\mathrm{C}_{17} \mathrm{H}_{28} \mathrm{O}_{6}$ & 329.1959 & 329.19595 & 15.5 & 0.34 \\
\hline 25 & $\mathrm{C}_{16} \mathrm{H}_{24} \mathrm{O}_{5}$ & 297.1697 & 297.16966 & 15.505 & 0.47 \\
\hline 26 & $\mathrm{C}_{16} \mathrm{H}_{33} \mathrm{NO}$ & 256.2635 & 256.26346 & 15.527 & 3.05 \\
\hline 27 & - & & 620.43719 & 15.555 & 0.73 \\
\hline 28 & $\mathrm{C}_{18} \mathrm{H}_{33} \mathrm{NO}_{2}$ & 296.2584 & 296.25842 & 15.591 & 3.05 \\
\hline 29 & - & & 576.41077 & 15.653 & 0.8 \\
\hline 30 & - & & 532.38476 & 15.748 & 0.77 \\
\hline 31 & - & & 488.35824 & 15.835 & 0.59 \\
\hline 32 & $\mathrm{C}_{20} \mathrm{H}_{34} \mathrm{~N}_{2} \mathrm{O}_{6}$ & 399.2490 & 399.25077 & 15.835 & 0.87 \\
\hline 33 & - & & 444.33208 & 15.895 & 0.38 \\
\hline 34 & $\mathrm{C}_{18} \mathrm{H}_{35} \mathrm{NO}$ & 282.2791 & 282.27922 & 16.023 & 9.17 \\
\hline 35 & $\mathrm{C}_{18} \mathrm{H}_{32} \mathrm{O}_{7}$ & 361.2221 & 361.22235 & 16.158 & 0.15 \\
\hline
\end{tabular}




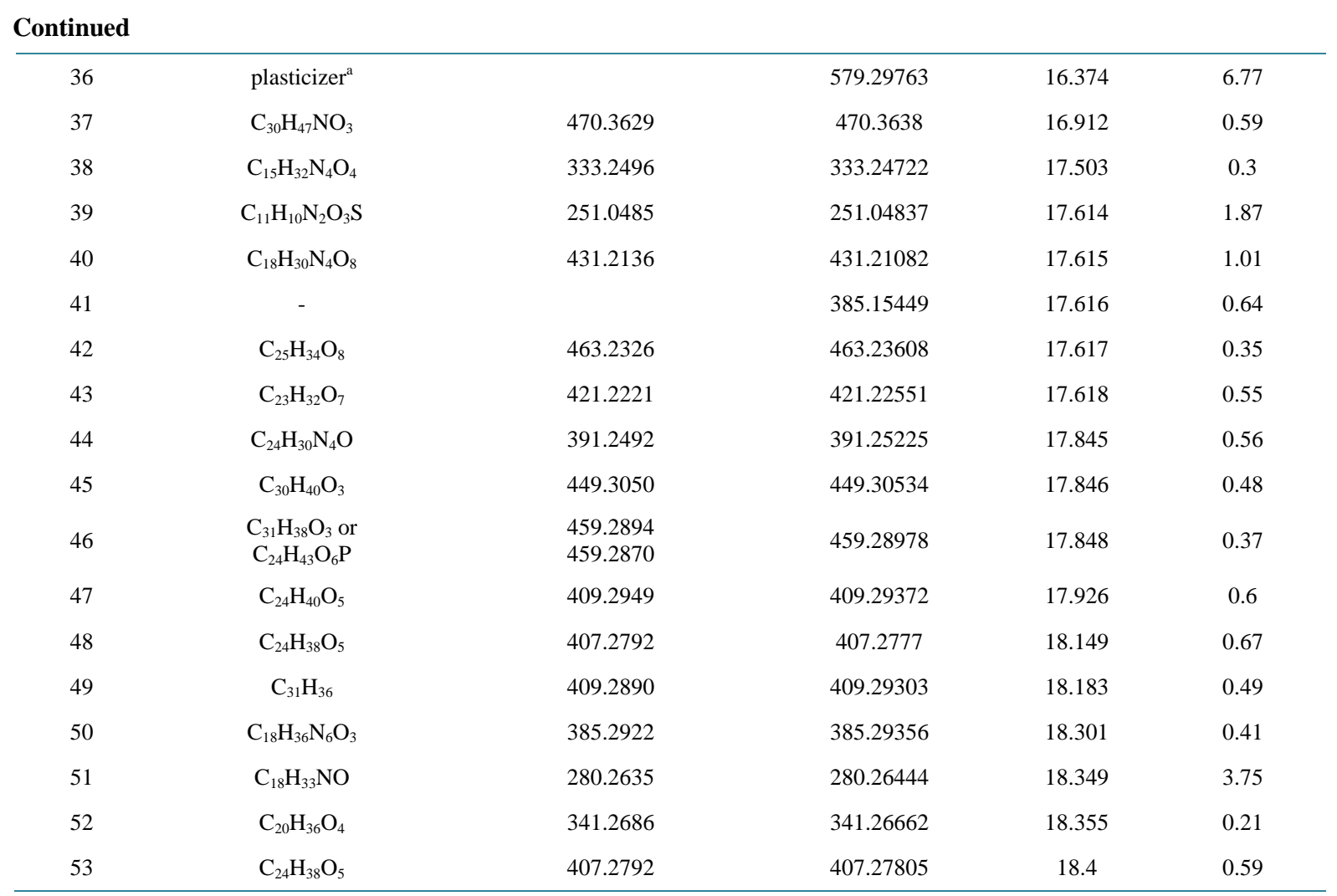

${ }^{\mathrm{a}}$ Ion peak of plasticizer dibutyl phthalate $[2 \mathrm{M}+\mathrm{Na}]^{+}$.

HPLC-TOF MS, ${ }^{1} \mathrm{H}$ - and ${ }^{13} \mathrm{C}$-NMR data with those reported. Their structures were identified as $\beta$-carboline (1) [6], 3-benzylhexahydropyrrolo[1,2-a]pyrazine-1,4-dione (2, cyclo-(Pro-Phe)) [7], and 3-isobutylhexahydropyrrolo[1,2-a]pyrazine-1,4-dione (3, cyclo-(Pro-Leu)) [7]. These purified compounds were alkaloids or cyclic dipeptides of simple structures, and more microbial material of Pseudomonas sp. NJ-011 should be provided for exploration of the minor constituents with complicated structures.

\section{Conclusion}

Diversity analysis on secondary metabolites by HPLC-TOF MS tests exhibited that the chemical constituents of Pseudomonas sp. NJ-011 were mainly N-containing compounds including some alkaloids and short polypeptides, while those of Rhodococcus sp. NJ-008 were not N-containing ones. One alkaloid and two cyclic dipeptides were also isolated and identified from extract of Pseudomonas sp. NJ-011, which confirmed the chemical diversity analysis. There have been more than 200 compounds reported from the Pseudomonas genus, but only a small part of them were from marine derived Pseudomonas spp. Some known cyclic dipeptides [8] and two new $\alpha$-pyrones [9] were reported recently, and more attention should be paid for chemical investigation on marine derived Pseudomonas spp.

\section{Acknowledgements}

We thank financial support from the National High Technology Research and Development Program (No. 2012AA092105), Chinese Polar Environment Comprehensive Investigation \& Assessment Programmes (CHINARE2014-01-06), and the Natural Science Foundation of Shanghai (11ZR1450000).

\section{References}

[1] Li, L.Y., Li, D.H., Luan, Y.P., Gu, Q.Q. and Zhu, T.J. (2012) Cytotoxic Metabolites from the Antarctic Psychrophilic Fungus Oidiodendron truncatum. Journal of Natural Products, 75, 920-927. http://dx.doi.org/10.1021/np3000443 
[2] Wu, G.W., Ma, H.Y., Zhu, T.J., Li, J., Gu, Q.Q. and Li, D.H. (2012) Penilactones A and B, Two Novel Polyketides from Antarctic Deep-Sea Derived Fungus Penicillium crustosum PRB-2. Tetrahedron, 68, 9745-9749. http://dx.doi.org/10.1016/j.tet.2012.09.038

[3] Ren, J.W., Xue, C.M., Tian, L., Xu, M.J., Chen, J., Deng, Z.W., Proksch, P. and Lin, W.H. (2009) Asperelines A-F, Peptaibols from the Marine-Derived Fungus Trichoderma asperellum. Journal of Natural Products, 72, 1036-1044.

[4] Ma, H.Y., Li, D.H., Gu, Q.Q. and Zhu, T.J. (2011) Bioactive Secondary Metabolites Produced by an Antarctic MarineDerived Fungus Penicillium chrysogenum PR4-1-3. Chinese Journal of Marine Drugs, 30, 18-24. http://dx.doi.org/10.1021/np900190w

[5] Ren, H., Cao, X.L. and Gu, Q.Q. (2010) Antitumor Metabolites from Marine-Derived Fungus Gliocladium Catenulatum T31. Chinese Pharmaceutical Journal, 45, 1720-1723.

[6] Kearns, P.S. and Rideout, J.A. (2008) Nonsymmetrical $\beta$-Carbolinedimers from an Ascidian, Didemnum sp. Journal of Natural Products, 71, 1280-1282. http://dx.doi.org/10.1021/np800077e

[7] Li, D.H., Gu, Q.Q., Zhu W.M., Liu, H.B., Fang, Y.C. and Zhu, T.J. (2005) Antitumor Components from Marine Actinomycete 11014 I: Cyclic Dipeptides. Chinese Journal of Antibiotics, 30, 449-452.

[8] Qi, S.H., Qian, P.Y. and Zhang, S. (2009) Antibacterial Metabolites from Marine Bacterium Pseudomonas sp. Natural Product Research and Development, 21, 420-423.

[9] Kong F.M., Singh, M.P. and Carter, G.T. (2005) Pseudopyronines A and B, $\alpha$-Pyrones Produced by a Marine Pseudomonas sp. F92S91, and Evidence for the Conversion of 4-Hydroxy- $\alpha$-pyrone to 3-Furanone. Journal of Natural Products, 68, 920-923. http://dx.doi.org/10.1021/np050038v 
Scientific Research Publishing (SCIRP) is one of the largest Open Access journal publishers. It is currently publishing more than 200 open access, online, peer-reviewed journals covering a wide range of academic disciplines. SCIRP serves the worldwide academic communities and contributes to the progress and application of science with its publication.

Other selected journals from SCIRP are listed as below. Submit your manuscript to us via either submit@scirp.org or Online Submission Portal.
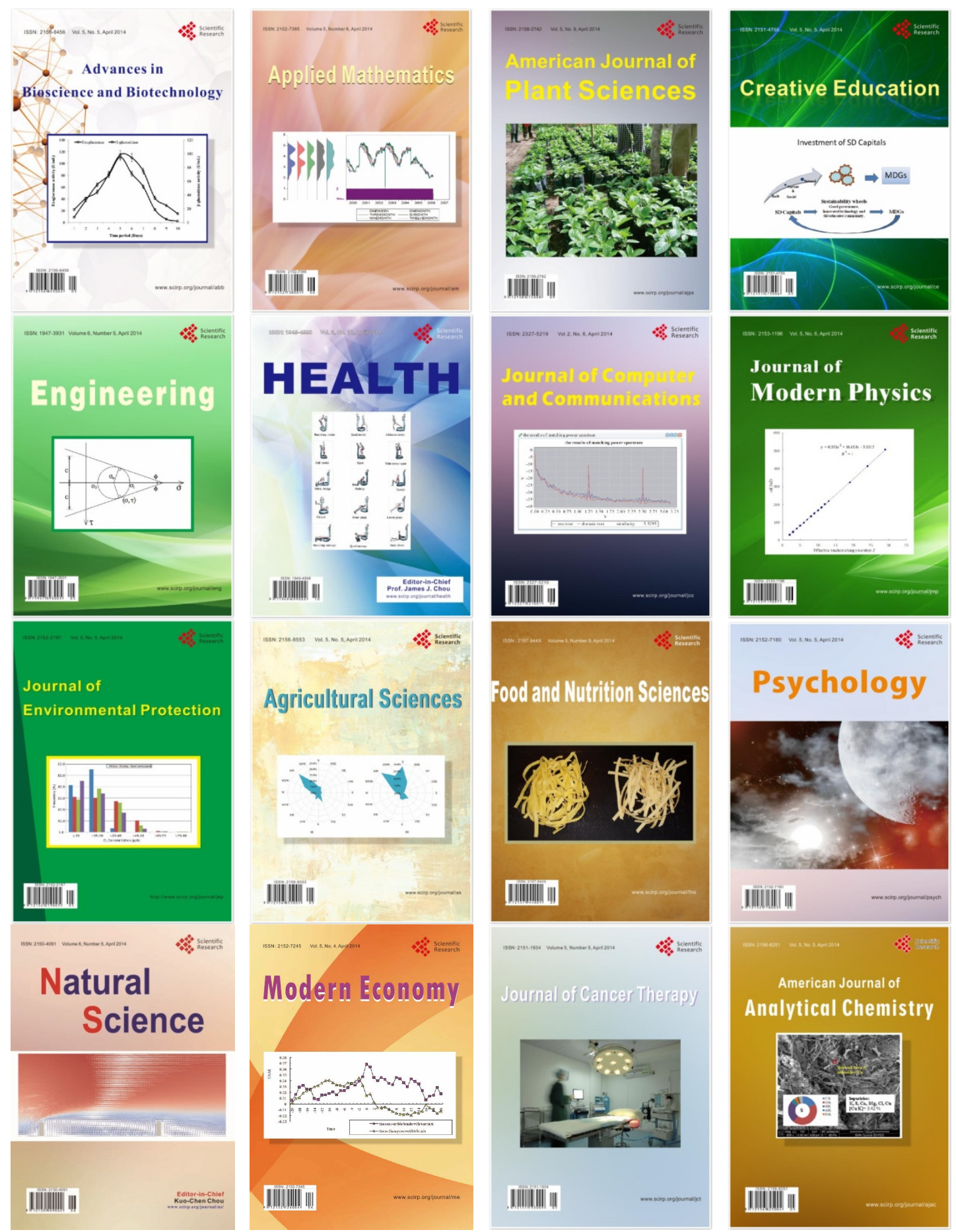